\title{
Electricity Production Scheduling under Uncertainty: Max Social Welfare vs. Min Emission vs. Max Renewable Production
}

\author{
Zhaowei Genga,*, Antonio J. Conejo ${ }^{\mathrm{b}}$, Qixin Chen $^{\mathrm{a}}$, Qing Xia ${ }^{\mathrm{a}}$, Chongqing Kang ${ }^{\mathrm{a}}$ \\ ${ }^{a}$ Department of Electrical Engineering, Tsinghua University, Beijing, 100084, China \\ ${ }^{b}$ Departments of ISE and ECE, The Ohio State University, Columbus, OH, 43202, USA
}

\begin{abstract}
Some areas in China are facing pressing air pollution problems. Measures from the power sector can be taken to cope with air pollution issues, including reducing emission levels of thermal units and integrating wind and solar power. Social welfare, emission, and renewable integration are three major concerns in modern power system operations. This paper describes three stochastic scheduling models aiming at maximizing social welfare (SW), minimizing emission (EM) and maximizing renewable production (RE). A multi-objective scheduling model (MT) is also proposed that properly balances the above objectives. Wind power uncertainty and dispatchable loads are considered in the model. The outcomes of the three models are compared through an illustrative example and a 57-node case study. Results show that model EM results in $36 \%$ of the social welfare of model SW, $27 \%$ of its emissions, and $43 \%$ of its wind spillage, while model RE results in $55 \%$ of the social welfare of model SW, $56 \%$ of its emissions and $28 \%$ of its wind spillage. Additionally, we analyze how the optimal generation scheduling is affected by the weights in model MT. This work provides insight to policy makers on how to balance social welfare, emissions and renewable production.
\end{abstract}

Keywords: Generation schedule, emission, renewable integration, social welfare, multi-objective

\section{Introduction}

\subsection{Motivation and Aim}

Some areas in China face significant air pollution problems. The power sector contributed $35 \%$ of $\mathrm{SO}_{2}$ emissions and 38\% of $\mathrm{NO}_{\mathrm{x}}$ emissions in 2014 [1]. Measures are being implemented in the power sector

5 to alleviate this air pollution problem. On one hand, the overall emission characteristics of thermal units have been greatly improved. On the other hand, the capacity of renewable energy, such as wind and solar power, has grown rapidly in the last decade.

Wind power has low marginal cost compared with thermal units, and involves neither air pollution emissions nor $\mathrm{CO}_{2}$ emissions. Wind power capacity is growing rapidly. However, the stochastic charac-

\footnotetext{
${ }^{*}$ Corresponding author. Tel.: +86-10-62770197

Email address: gengzw12@mails.tsinghua.edu.cn (Zhaowei Geng)
} 
10 production in China in 2015 [2].

Besides deployment of up/down reserves by thermal units, dispatchable loads provide an appropriate mechanism to deal with uncertainty. Dispatchable loads can be scheduled and re-dispatched like generators to achieve the scheduling objective. Dispatchable loads increase the flexibility of the system, which is helpful 15 in system with high renewable production.

To minimize the operating cost is the main objective for most Independent System Operators (ISO), but nowadays other objectives appear. For some highly-polluted areas, the ISO may seek to minimize emissions to ensure air quality. For areas with high renewable integration, the ISO may seek to maximize renewable production to make electricity production as "green" as possible. These distinct objectives result 20 in different generation scheduling outcomes.

Considering the pressing problems of air pollution and wind curtailment in China, this paper focuses on the comparison of three stochastic scheduling models taking into account wind power uncertainty and dispatchable loads. The objectives are to maximize social welfare, to minimize emissions, and to maximize renewable integration, respectively. A multi-objective scheduling model that properly combines the above An illustrative example and a 57-node case study are considered to illustrate the proposed models.

\subsection{Literature Review}

Relevant works pertaining to electricity generation scheduling models with different objectives are reviewed below. Minimizing cost (or maximizing social welfare) is the most common goal [3, 4] in unit the cost considering system reserves, and proposes a two-stage optimization method to solve the model. Wang et al. [4] proposes a security-constrained unit commitment model that minimizes cost considering the volatility of wind power generation. Jiang et al. [5] compares maximizing social welfare and minimizing cost in a day-ahead wholesale electricity market considering demand side management. Gent and Lamont

35 [6] propose a dispatch model to minimize $\mathrm{NO}_{\mathrm{x}}$ emissions. An optimal power flow model to minimize wind spillage is proposed in [7].

To deal with multiple targets in power system scheduling, multi-objective optimization models are used. Many works co-optimize cost and emission in deterministic or stochastic unit commitment problems $[8,9,10,11,12]$. Glotic and Zamuda [8] compare minimizing fuel cost and minimizing emissions in a 40 hydro thermal power system, and formulate a decomposition model to co-optimize these two objectives together. In [9] and [10], similar power generation scheduling models with thermal and hydro units are formulated, and the cost vs. emission curve are provided using Pareto optimality. Sadeghian et al. [11] builds a generation scheduling model with the objective of maximizing profit and minimizing emissions considering heat and electricity supply. A stochastic model with the goal of cost and emission minimization 
that considers the uncertainty of wind power and photovoltaic units and market price is proposed in [12]. References [13] and [14] minimize cost and emissions in generation scheduling problems considering wind power uncertainty and demand response. Prebeg et al. [15] focus on the long-term energy planning of the Croatian power system with renewable energy and electric vehicles, and considers the objectives of minimizing the net present value, minimizing the net present value divided by the energy produced, and maximizing the renewable energy production.

Note that generally these objectives conflict with each other and cannot be optimized simultaneously. A variety of methodologies such as the weighted sum method [11], goal programming [16, 17], and $\epsilon$ constrained method $[9,18]$ can be used to solve multi-objective optimization problems. In the weighted sum method, the decision maker states the relative importance of each objective function with weight

55 factors and then add the weighted objective functions together. In goal programming, the targets are set for all the objectives, and the idea is to minimize the deviation from the goals. In the $\epsilon$-constrained method, one objective is selected, and the other objectives are converted into constraints. Recently, Charitopoulos and Dua [18] propose a unified framework for multi-objective energy optimization considering uncertain parameters, and develop an algorithm based on the $\epsilon$-constrained method to solve it. Trivedi et al. [19]

60 formulates a multi-objective day-ahead thermal generation scheduling model that considers operation cost, emission cost, loss of load probability and expected unserved energy, as objective functions or constraints.

In some works, specific optimal results are not obtained directly, but a Pareto-optimal frontier (or Pareto-optimal set) is obtained [10, 13, 20] instead. Li and Qiu [20] consider a system with hydro power and photovoltaic power and seek to minimize the variance of power output and/or maximizing total energy generated by this renewable energy production system. The Pareto-optimal set is obtained using a modified version of a non-dominated sorting genetic algorithm [21]. Fuzzy decision-making is a methodology used to find the final optimal solution considering the decision maker's preferences about the objectives after a Pareto optimal solution set is obtained [13, 22]. Hozouri et al. [22] uses a fuzzy decision-making method to balance minimum wind energy curtailment, minimum social cost, and maximum energy storage revenue.

Finally, we note that since wind power is stochastic, stochastic unit commitment is generally used in systems with important wind power uncertainty [23, 24, 25, 26, 27].

\subsection{Contributions}

Contributions of this paper are threefold:

1. To propose a versatile two-stage stochastic scheduling model considering wind power uncertainty and dispatchable loads pursuing three objectives: maximum social welfare, minimum emissions, and maximum renewable energy integration.

2. To formulate a comprehensive multi-objective scheduling model that combines these three objectives.

3. To analyze the generation scheduling results under different government policies. 
Note that the novelty of the proposed analysis regarding the interactions of cost vs. emissions vs.

80 wable-integration in systems with demand flexibility and important renewable penetration. Moreover, we use a rigorous two-stage stochastic programming modeling framework.

\subsection{Paper Organization}

This paper is organized as follows. Section 2 formulates the stochastic scheduling problems with the three different objectives and a parameterized combination of these objectives. Case studies are provided 85 in section 3. Conclusions are given in section 4 .

\section{Model Formulation}

In this section, the three single-objective models and the multi-objective model are presented. They follow the model structure proposed in $[28,29,30]$.

\subsection{Notation}

90

\section{Indexes:}

$i$ Index of thermal units

$j$ Index of demands

$n$ Index of nodes

$p$ Index of air pollutants, such as $\mathrm{SO}_{2}$ and $\mathrm{NO}_{\mathrm{x}}$

95

$s$ Index of scenarios

$t$ Index of time periods

$w$ Index of wind units

\section{Sets:}

$T$ Set of time periods

100

$\Omega^{\mathrm{N}}$ Set of nodes

$\Omega_{n}^{\mathrm{N}}$ Set of nodes adjacent to node $n$

$\Omega^{\mathrm{I}}$ Set of thermal units

$\Omega_{n}^{\mathrm{I}}$ Set of thermal units at node $n$

$\Omega^{\mathrm{J}}$ Set of demands

${ }_{105} \Omega_{n}^{\mathrm{J}}$ Set of demands at node $n$ 
$\Omega^{\mathrm{L}}$ Set of transmission lines

$\Omega^{\mathrm{P}}$ Set of air pollutants

$\Omega^{\mathrm{S}}$ Set of scenarios

$\Omega^{\mathrm{W}}$ Set of wind units

110

$\Omega_{n}^{\mathrm{W}}$ Set of wind units at node $n$

\section{Variables:}

$z_{i t}$ On/off status of unit $i$ at time $t: 1$ if on, 0 if off

$y_{i t}^{\mathrm{SD}}$ Shut-down indicator of unit $i$ at time $t$ : 1 if shut-down at the beginning of period $t, 0$ otherwise

$y_{i t}^{\mathrm{SU}}$ Start-up indicator of unit $i$ at time $t$ : 1 if started-up at the beginning of period $t, 0$ otherwise

$D_{j t}$ Scheduled load of demand $j$ at time $t$

$D_{j t s}$ Load of demand $j$ at time $t$ in scenario $s$

$D_{j t s}^{\text {SHED }}$ Load shedding of demand $j$ at time $t$ in scenario $s$

$d_{j t s}^{\mathrm{D}}$ Load decrease of dispatchable demand $j$ during period $t$ and scenario $s$

$d_{j t s}^{\mathrm{U}}$ Load increase of dispatchable demand $j$ during period $t$ and scenario $s$

$P_{i t}$ Scheduled power output of unit $i$ at time $t$

$P_{i t s}$ Power output of unit $i$ at time $t$ in scenario $s$

$r_{i t s}^{\mathrm{D}}$ Down-reserve deployment of unit $i$ at time $t$ in scenario $s$ to comply with wind uncertainty realization

$r_{i t s}^{\mathrm{U}}$ Up-reserve deployment of unit $i$ at time $t$ in scenario $s$ to comply with wind uncertainty realization

$W_{w t}$ Scheduled power output of wind unit $w$ at time $t$

$W_{w t s}^{\mathrm{SP}}$ Power spillage of wind unit $w$ at time $t$ in scenario $s$

$\theta_{n t}$ Voltage angle of node $n$ at time $t$ at the scheduling stage

$\theta_{n t s}$ Voltage angle of node $n$ at time $t$ in scenario $s$

\section{Parameters:}

$C_{i}$ Marginal cost for unit $i, \$ / \mathrm{MWh}$

$C_{w}$ Cost for wind unit $w, \$ / \mathrm{MWh}$ 
$C_{w}^{\mathrm{WSP}}$ "Cost" for wind spillage for unit $w, \$ / \mathrm{MWh}$

$C_{p}^{\mathrm{E}}$ Emission fee of pollutant $p, \$ / \mathrm{kg}$

$D_{j t}^{\max }$ Maximum load of demand $j$ at time $t, \mathrm{MW}$

$D_{j t}^{\min }$ Minimum load of demand $j$ at time $t, \mathrm{MW}$

$E_{j}^{\text {min }}$ Minimum energy supplied of demand $j$ during the scheduling period, MWh

$F_{n r}^{\max }$ Transmission capacity of line $n-r, \mathrm{MW}$

$K_{j}^{\mathrm{D}, \max }$ Ramp down limit for demand $j, \mathrm{MW}$

$K_{j}^{\mathrm{U}, \max }$ Ramp up limit for demand $j$, MW

${ }_{140} L_{i}^{\mathrm{D}, \max }$ Ramp down limit for unit $i$, MW

$L_{i}^{\mathrm{U}, \max }$ Ramp up limit for unit $i$, MW

$P_{i}^{\max }$ Capacity of unit $i, \mathrm{MW}$

$P_{i}^{\min }$ Minimum power output of unit $i$, MW

$R_{i}^{\mathrm{D}, \max }$ Maximum down reserve for unit $i, \mathrm{MW}$

${ }_{145} R_{i}^{\mathrm{U}, \max }$ Maximum up reserve for unit $i, \mathrm{MW}$

$U_{j t}$ Marginal utility of demand $j, \$ / \mathrm{MWh}$

$V_{j}^{\mathrm{LOL}}$ Value of loss of load for demand $j, \$ / \mathrm{MWh}$

$W_{w t s}$ Wind power forecast of wind $w$ at time $t$ in scenario $s, \mathrm{MW}$

$w_{f}$ Weight of the conflicting objectives

$x_{n r}$ Per-unit reactance of line $n-r$

$\pi_{i}^{\mathrm{SU}}$ Start-up cost of unit $i, \$$

$\rho_{s}$ Probability of scenario $s$

$\sigma_{i}^{p}$ Emission factor of pollutant $p$ for unit $i, \mathrm{~kg} / \mathrm{MWh}$

$\sigma_{i}^{\mathrm{SU}, p}$ Start-up emission of pollutant $p$ for unit $i, \mathrm{~kg}$ 
The proposed model is based on the following assumptions:

1. Wind power production is stochastic and represented by a set of possible scenarios.

2. Load is dispatchable within a given range. The range is deterministic.

3. For each demand, there is a minimum energy consumption per day that should be guaranteed.

4. The marginal utility of every demand, which changes with time, is data.

5. The cost of up/down reserve deployment for thermal units is the same as the cost of energy production.

6. The transmission network is represented using a dc model.

\subsection{Formulation}

To deal with the stochastic nature of wind power production, the scheduling problem is formulated as a two-stage stochastic programming model. At the scheduling stage, the on/off status and the scheduling power output of units, and the scheduling load, are decided. At the operation stage, the power output of units and the loads are adjusted according to wind power realization in each scenario. Detailed formulation is provided below.

\subsubsection{Objectives}

${ }_{170}$ We consider three possible objectives:

1. Minimum minus social welfare (maximum social welfare)

$$
\begin{array}{r}
\min _{\Xi} f_{1}=\sum_{t \in T} \sum_{i \in \Omega^{\mathrm{I}}} \pi_{i}^{\mathrm{SU}} y_{i t}^{\mathrm{SU}}+\sum_{t \in T} \sum_{s \in \Omega^{\mathrm{S}}} \rho_{s}\left[\sum_{i \in \Omega^{\mathrm{I}}} C_{i} P_{i t s}\right. \\
\left.+\sum_{w \in \Omega^{\mathrm{W}}} C_{w}\left(W_{w t s}-W_{w t s}^{\mathrm{SP}}\right)-\sum_{j \in \Omega^{\mathrm{J}}} U_{j t} D_{j t s}+\sum_{j \in \Omega^{\mathrm{J}}} V_{j}^{\mathrm{LOL}} D_{j t s}^{\mathrm{SHED}}\right]
\end{array}
$$

2. Minimum emissions

$$
\min _{\Xi} f_{2}=\sum_{t \in T} \sum_{p \in \Omega^{\mathrm{P}}} \sum_{i \in \Omega^{\mathrm{I}}} C_{p}^{\mathrm{E}} \sigma_{i}^{\mathrm{SU}, p} y_{i t}^{\mathrm{SU}}+\sum_{s \in \Omega^{\mathrm{S}}} \rho_{s} \sum_{t \in T}\left[\sum_{p \in \Omega^{\mathrm{P}}} \sum_{i \in \Omega^{\mathrm{I}}} C_{p}^{\mathrm{E}} \sigma_{i}^{p} P_{i t s}+\sum_{j \in \Omega^{\mathrm{J}}} V_{j}^{\mathrm{LOL}} D_{j t s}^{\mathrm{SHED}}\right]
$$

3. Minimum wind spillage (maximum renewable integration)

$$
\min _{\Xi} f_{3}=\sum_{s \in \Omega^{\mathrm{S}}} \rho_{s} \sum_{t \in T}\left[\sum_{w \in \Omega^{\mathrm{W}}} C_{w}^{\mathrm{WSP}} W_{w t s}^{\mathrm{SP}}+\sum_{j \in \Omega^{\mathrm{J}}} V_{j}^{\mathrm{LOL}} D_{j t s}^{\mathrm{SHED}}\right]
$$

Cost for generator start-up and operation and the utility of demand are considered in objective (1). The social welfare is defined as the total utility of loads minus the total cost of generation and the cost of load shedding [5, 31, 32]. $f_{1}$ in Equation (1) is the minus social welfare to be minimized. Cost for generator 
start-up and operation emissions is considered in objective (2). Cost for wind spillage is considered in

objective (3). Load shedding cost is considered in every objective. Since each objective to be minimized is in dollars, the objective function of the multi-objective model is represented by the convex combination of the three single-objectives.

$$
\min _{\Xi} w_{1} f_{1}+w_{2} f_{2}+w_{3} f_{3}
$$

where $w_{1}+w_{2}+w_{3}=1, w_{f} \geq 0, f=1,2,3$.

\subsubsection{Constraints at Scheduling Stage}

$$
\begin{aligned}
& z_{i, t-1}+y_{i t}^{\mathrm{SU}}-y_{i t}^{\mathrm{SD}}-z_{i t}=0, \forall i \in \Omega^{\mathrm{I}}, \forall t \in T \\
& y_{i t}^{\mathrm{SU}}+y_{i t}^{\mathrm{SD}} \leq 1, \quad \forall i \in \Omega^{\mathrm{I}}, \forall t \in T \\
& \sum_{i \in \Omega_{n}^{\mathrm{I}}} P_{i t}+\sum_{w \in \Omega_{n}^{\mathrm{W}}} W_{w t}-\sum_{j \in \Omega_{n}^{\mathrm{J}}} D_{j t}+\sum_{r \in \Omega_{n}^{\mathrm{N}}} \frac{1}{x_{n r}}\left(\theta_{r t}-\theta_{n t}\right)=0, \quad \forall n \in \Omega^{\mathrm{N}}, \forall t \in T \\
& z_{i t} P_{i}^{\mathrm{min}} \leq P_{i t} \leq z_{i t} P_{i}^{\max }, \quad \forall i \in \Omega^{\mathrm{I}}, \forall t \in T \\
& \sum_{t \in T} D_{j t} \geq E_{j}^{\mathrm{min}}, \quad \forall j \in \Omega^{J} \\
& \theta_{\mathrm{ref}, t}=0, \quad \forall t \in T \\
& z_{i t}, y_{i t}^{\mathrm{SU}}, y_{i t}^{\mathrm{SD}} \in\{0,1\}, \quad \forall i \in \Omega^{\mathrm{I}}, \forall t \in T
\end{aligned}
$$

Constraints (5a) and (5b) model on/off status changes. Constraint (5c) enforces the power balance at each node. Constraint (5d) enforces the power output range of production units. Constraint (5e) regulates the energy consumption level. Constraint (5f) defines the reference angle. Constraint (5g) specifies binary variables.

2.3.3. Constraints at Operation Stage

$$
\begin{gathered}
\sum_{i \in \Omega_{n}^{\mathrm{I}}}\left(r_{i t s}^{\mathrm{U}}-r_{i t s}^{\mathrm{D}}\right)+\sum_{w \in \Omega_{n}^{\mathrm{W}}}\left(W_{w t s}-W_{w t}-W_{w t s}^{\mathrm{SP}}\right)-\sum_{j \in \Omega_{n}^{\mathrm{J}}}\left(d_{j t s}^{\mathrm{U}}-d_{j t s}^{\mathrm{D}}-D_{j t s}^{\mathrm{SHED}}\right) \\
+\sum_{r \in \Omega_{n}^{\mathrm{N}}} \frac{1}{x_{n r}}\left[\left(\theta_{r t s}-\theta_{n t s}\right)-\left(\theta_{r t}-\theta_{n t}\right)\right]=0, \quad \forall n \in \Omega^{\mathrm{N}}, \forall t \in T, \forall s \in \Omega^{\mathrm{S}} \\
z_{i t} P_{i}^{\min } \leq P_{i t s} \leq z_{i t} P_{i}^{\max }, \quad \forall i \in \Omega^{\mathrm{I}}, \forall t \in T, \forall s \in \Omega^{\mathrm{S}} \\
-L_{i}^{\mathrm{D}, \max } \leq P_{i t s}-P_{i, t-1, s} \leq L_{i}^{\mathrm{U}, \max }, \quad \forall i \in \Omega^{\mathrm{I}}, \forall t \in T, \forall s \in \Omega^{\mathrm{S}} \\
-K_{j}^{\mathrm{D}, \max } \leq D_{j t s}-D_{j, t-1, s} \leq K_{j}^{\mathrm{U}, \max }, \quad \forall j \in \Omega^{\mathrm{J}}, \forall t \in T, \forall s \in \Omega^{\mathrm{S}} \\
\sum_{t \in T} D_{j t s} \geq E_{j}^{\min }, \quad \forall j \in \Omega^{J}, \forall s \in \Omega^{S}
\end{gathered}
$$




$$
\begin{aligned}
-F_{n r}^{\max } \leq \frac{1}{x_{n r}}\left(\theta_{n t s}-\theta_{r t s}\right) \leq F_{n r}^{\max }, & \forall(n, r) \in \Omega^{\mathrm{L}}, \forall t \in T, \forall s \in \Omega^{\mathrm{S}} \\
\theta_{\text {ref }, t s}=0, & \forall t \in T, \forall s \in \Omega^{\mathrm{S}} \\
D_{j t}^{\mathrm{min}} \leq D_{j t s} \leq D_{j t}^{\max }, & \forall j \in \Omega^{\mathrm{J}}, \forall t \in T, \forall s \in \Omega^{\mathrm{S}} \\
0 \leq D_{j t s}^{\mathrm{SHED}} \leq D_{j t}, & \forall j \in \Omega^{\mathrm{J}}, \forall t \in T, \forall s \in \Omega^{\mathrm{S}} \\
0 \leq W_{w t s}^{\mathrm{SP}} \leq W_{w t s}, & \forall w \in \Omega^{\mathrm{W}}, \forall t \in T, \forall s \in \Omega^{\mathrm{S}} \\
0 \leq r_{i t s}^{\mathrm{U}} \leq R_{i}^{\mathrm{U}, \max }, & \forall i \in \Omega^{\mathrm{I}}, \forall t \in T, \forall s \in \Omega^{\mathrm{S}} \\
0 \leq r_{i t s}^{\mathrm{D}} \leq R_{i}^{\mathrm{D}, \max }, & \forall i \in \Omega^{\mathrm{I}}, \forall t \in T, \forall s \in \Omega^{\mathrm{S}}
\end{aligned}
$$

Constraint (6a) is the power balance constraint. Constraint (6b) enforces the power output feasible ranges. Constraint (6c) imposes ramping limits for units. Constraint (6d) imposes ramping limits for loads. The minimum daily supplied energy $E_{j}^{\mathrm{min}}$ is enforced by constraint (6e). Constraint (6f) enforces transmission limits. Constraint $(6 \mathrm{~g})$ defines the reference angle for each operating condition. Constraints (6i)-(6l) impose bounds on load shedding, wind spillage and reserve deployment.

\subsubsection{Linking Constraints}

$$
\begin{gathered}
P_{i t s}=P_{i t}+r_{i t s}^{\mathrm{U}}-r_{i t s}^{\mathrm{D}}, \quad \forall i \in \Omega^{\mathrm{I}}, \forall t \in T, \forall s \in \Omega^{\mathrm{S}} \\
D_{j t s}=D_{j t}+d_{j t s}^{\mathrm{U}}-d_{j t s}^{\mathrm{D}}, \quad \forall j \in \Omega^{\mathrm{J}}, \forall t \in T, \forall s \in \Omega^{\mathrm{S}}
\end{gathered}
$$

Constraint (7) links generator power outputs and load at operation and scheduling stages.

The set of the decision variables is $\boldsymbol{\Xi}=\left\{z_{i t}, y_{i t}^{\mathrm{SU}}, y_{i t}^{\mathrm{SD}}, P_{i t}, P_{i t s}, r_{i t s}^{\mathrm{U}}, r_{i t s}^{\mathrm{D}}, W_{w t}, W_{w t s}^{\mathrm{SP}}, D_{j t}, D_{j t s}, D_{j t s}^{\mathrm{SHED}}\right.$, $\left.d_{j t s}^{\mathrm{U}}, d_{j t s}^{\mathrm{D}}, \theta_{n t}, \theta_{n t s}\right\}$.

\subsection{Computational Complexity}

Table 1: Size of formulation

\begin{tabular}{lc}
\hline & Number \\
\hline Binary Vars. & $3\left|\Omega^{\mathrm{I}}\right||T|$ \\
Continuous Vars. & $\left(1+3\left|\Omega^{\mathrm{S}}\right|\right)\left|\Omega^{\mathrm{I}}\right||T|+\left(1+\left|\Omega^{\mathrm{S}}\right|\right)\left(\left|\Omega^{\mathrm{N}}\right|+\left|\Omega^{\mathrm{W}}\right|\right)|T|+\left(1+4\left|\Omega^{\mathrm{S}}\right|\right)\left|\Omega^{\mathrm{J}}\right||T|$ \\
\hline Scheduling Cons. & $\left(4\left|\Omega^{\mathrm{I}}\right|+\left|\Omega^{\mathrm{N}}\right|\right)|T|+\left|\Omega^{\mathrm{J}}\right|$ \\
Operation Cons. & $\left(\left|\Omega^{\mathrm{N}}\right|+4\left|\Omega^{\mathrm{I}}\right|+2\left|\Omega^{\mathrm{J}}\right|+\left|\Omega^{\mathrm{L}}\right|\right)|T|\left|\Omega^{\mathrm{S}}\right|+\left|\Omega^{\mathrm{J}}\right|\left|\Omega^{\mathrm{S}}\right|$ \\
Linking Cons. & $\left(\left|\Omega^{\mathrm{I}}\right|+\left|\Omega^{\mathrm{J}}\right|\right)|T|\left|\Omega^{\mathrm{S}}\right|$ \\
\hline
\end{tabular}

Problem (1)-(7) is a Mixed-integer Linear Programming (MILP) problem. The size of this problem is provided in Table 1, where $|\Omega|$ represents the cardinality of set $\Omega$. This problem can be solved using standard optimization software, such as CPLEX [33]. 


\section{Case Studies}

To test the proposed model, an illustrative 2-node example and a 57-node case study are considered in this section.

\subsection{Illustrative Example}

\subsubsection{Data}

First we consider the 2-node example shown in Fig. 1. This example includes 3 time periods and 2 scenarios. Only $\mathrm{SO}_{2}$ emissions are considered. Generation parameters are provided in Table 2. Load ranges and utilities are provided in Table 3. The value of loss of load is $\$ 200 / \mathrm{MWh}$. There are two scenarios with probabilities 0.6 and 0.4 , respectively. Scenario $s 1$ has higher wind production than scenario $s 2$, as provided in Table 4. Costs of wind power and wind spillage are $\$ 3 / \mathrm{MWh}$. Emission cost for $\mathrm{SO}_{2}$ is $\$ 1 / \mathrm{kg}$. The transmission line has a reactance of 0.13 p.u., and a capacity of $50 \mathrm{MW}$. The three models are referred to as SW (maximum social welfare), EM (minimum emission) and RE (maximum renewable energy). The comprehensive model is referred to as MT.

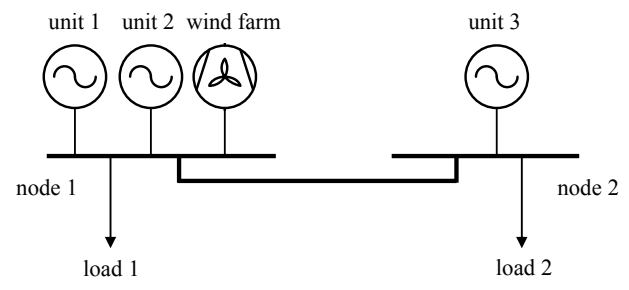

Figure 1: 2-node system.

Table 2: Unit parameters

\begin{tabular}{cccc}
\hline Unit & $i 1$ & $i 2$ & $i 3$ \\
\hline$P_{i}^{\max }(\mathrm{MW})$ & 50 & 110 & 100 \\
$P_{i}^{\min }(\mathrm{MW})$ & 10 & 10 & 5 \\
$C_{i}(\$ / \mathrm{MWh})$ & 10 & 30 & 35 \\
$R_{i}^{\mathrm{U}, \max }(\mathrm{MW})$ & 10 & 22 & 20 \\
$R_{i}^{\mathrm{D}, \max }(\mathrm{MW})$ & 10 & 22 & 20 \\
$\pi_{i}^{\mathrm{SU}}(\$)$ & 0 & 0 & 0 \\
$\sigma_{i}^{\mathrm{SO}}(\$ / \mathrm{kg})$ & 8 & 12 & 3 \\
$L_{i}^{\mathrm{U}, \max }(\mathrm{MW} / \mathrm{h})$ & 10 & 20 & 60 \\
$L_{i}^{\mathrm{U}, \max }(\mathrm{MW} / \mathrm{h})$ & 10 & 20 & 60 \\
\hline
\end{tabular}


Table 3: Load parameters (MW)

\begin{tabular}{ccc|lc}
\hline & \multicolumn{2}{c}{ Load Range(MW) } & \multicolumn{2}{|l}{ Marginal Utility (\$/MWh) } \\
\cline { 2 - 5 } & $D_{1}$ & $D_{2}$ & $D_{1}$ & $D_{2}$ \\
\hline$t 1$ & $32-48$ & $80-120$ & 40 & 15 \\
$t 2$ & $20-30$ & $64-96$ & 25 & 30 \\
$t 3$ & $36-54$ & $76-114$ & 35 & 40 \\
\hline
\end{tabular}

Table 4: Wind power forecast (MW)

\begin{tabular}{ccc} 
& $s 1$ High & $s 2$ Low \\
\hline$t 1$ & 50 & 10 \\
$t 2$ & 75 & 30 \\
$t 3$ & 35 & 15 \\
\hline
\end{tabular}

Table 5: Total cost, emissions, wind spillage and load shedding using each model

\begin{tabular}{|c|c|c|c|c|c|c|c|c|}
\hline & Model & $\begin{array}{c}\text { Social Welfare } \\
(\$)\end{array}$ & $\begin{array}{l}\text { Demand Utility } \\
\qquad \$()\end{array}$ & $\begin{array}{l}\text { Cost } \\
(\$)\end{array}$ & $\begin{array}{c}\text { Emissions } \\
(\mathrm{kg})\end{array}$ & $\begin{array}{c}\text { Wind Spillage } \\
\text { (MWh) }\end{array}$ & $\begin{array}{c}\text { Demand Supplied } \\
\text { (MWh) }\end{array}$ & $\begin{array}{c}\text { Load Shedding } \\
\text { (MWh) }\end{array}$ \\
\hline \multirow{4}{*}{ exp. } & SW & 5,261 & 12,588 & 7,327 & 1,806 & 21 & 403 & 0 \\
\hline & EM & 2,560 & 11,465 & 8,905 & 988 & 3 & 385 & 0 \\
\hline & $\mathrm{RE}$ & 2,339 & 11,969 & 9,630 & 1,198 & 0 & 395 & 0 \\
\hline & MT & 5.142 & 12,105 & 6,963 & 1,574 & 20 & 389 & 0 \\
\hline \multirow{4}{*}{$s 1$} & SW & 6,035 & 12,840 & 6,805 & 1,716 & 35 & 410 & 0 \\
\hline & EM & 3,685 & 11,450 & 7,765 & 840 & 5 & 385 & 0 \\
\hline & $\mathrm{RE}$ & 3,825 & 12,365 & 8,540 & 1,064 & 0 & 402 & 0 \\
\hline & MT & 5,904 & 12,105 & 6,201 & 1,456 & 33 & 389 & 0 \\
\hline \multirow{4}{*}{$s 2$} & SW & 4,100 & 12,210 & 8,110 & 1,941 & 0 & 392 & 0 \\
\hline & EM & 835 & 11,450 & 10,615 & 1,210 & 0 & 385 & 0 \\
\hline & $\mathrm{RE}$ & 110 & 11,375 & 11,265 & 1,400 & 0 & 385 & 0 \\
\hline & MT & 4,000 & 12,105 & 8,105 & 1,752 & 0 & 389 & 0 \\
\hline
\end{tabular}

\subsubsection{Results}

The total expected and per scenario social welfare, demand utility, cost, emission, wind spillage, demand supplied, and load shedding are provided in Table 5. Model SW results in the highest social welfare (total demand utility minus total expected cost), but highest emissions and wind spillage among all the models.

The higher wind scenario results in lower cost under each model. However, wind integration can be expensive, as we can see from the high cost under model RE. Model MT, with weights $(0.2,0.3,0.5)$, provides intermediate results in social welfare, emissions and wind spillage, but the lowest cost.

The power outputs from each model are provided in Table 6. Power flows for the four models are provided in Table 7 . The most polluting unit, i2, is not committed if model EM is used. Although expensive, unit $i 3$ is committed even if model $\mathrm{SW}$ is used due to the congestion in the transmission line. 
The lowest load and highest wind happen simultaneously at time $t 2$, when wind spillage appears as provided by Table 9 . Under model RE, unit $i 1$ and $i 2$ are both shut down at time $t 2$ to accommodate wind power at the same node.

Results of load at the scheduling and operation levels from each model are provided in Table 8. Generally, load flexibility helps. Loads are high at nodes where the marginal utility is higher than the marginal cost if model SW is used. If model EM is used, the peak load tends to be curtailed and the valley load tends to increase, to reduce the peak emissions during peak load. If model RE is used, loads increases to their maximum to accommodate increasing amounts of wind power until wind spillage drops to zero.

Table 6: Power outputs from each model(MW)

\begin{tabular}{|c|c|c|c|c|c|c|c|c|c|c|}
\hline & \multirow{3}{*}{ Time } & \multicolumn{3}{|c|}{ Schedule } & \multicolumn{6}{|c|}{ Operation } \\
\hline & & \multirow{2}{*}{$i 1$} & \multirow{2}{*}{$i 2$} & \multirow{2}{*}{$i 3$} & \multicolumn{2}{|c|}{$i 1$} & \multicolumn{2}{|c|}{$i 2$} & \multicolumn{2}{|c|}{$i 3$} \\
\hline & & & & & $s 1$ & $s 2$ & $s 1$ & $s 2$ & $s 1$ & $s 2$ \\
\hline \multirow{3}{*}{ SW } & $t 1$ & 48 & 0 & 48 & 48 & 50 & 0 & 0 & 30 & 68 \\
\hline & $t 2$ & 40 & 0 & 30 & 40 & 50 & 0 & 0 & 34 & 34 \\
\hline & $t 3$ & 50 & 19 & 64 & 50 & 50 & 19 & 20 & 64 & 65 \\
\hline \multirow{3}{*}{ EM } & $t 1$ & 14 & 0 & 84 & 10 & 14 & 0 & 0 & 64 & 100 \\
\hline & $t 2$ & 10 & 0 & 66 & 10 & 10 & 0 & 0 & 46 & 86 \\
\hline & $t 3$ & 10 & 0 & 100 & 10 & 20 & 0 & 0 & 90 & 100 \\
\hline \multirow{3}{*}{$\mathrm{RE}$} & $t 1$ & 10 & 20 & 69 & 10 & 10 & 20 & 20 & 49 & 89 \\
\hline & $t 2$ & 0 & 0 & 71 & 0 & 0 & 0 & 0 & 51 & 91 \\
\hline & $t 3$ & 0 & 12 & 100 & 0 & 0 & 12 & 20 & 100 & 100 \\
\hline \multirow{3}{*}{$\mathrm{MT}$} & $t 1$ & 48 & 0 & 48 & 48 & 50 & 0 & 0 & 30 & 68 \\
\hline & $t 2$ & 40 & 0 & 32.5 & 38 & 50 & 0 & 0 & 32.5 & 32.5 \\
\hline & $t 3$ & 48 & 0 & 65.5 & 48 & 50 & 0 & 0 & 65.5 & 83.5 \\
\hline
\end{tabular}

Table 7: Power flow from $n 1$ to $n 2$ (MW)

\begin{tabular}{ccc|cc|cc|cc}
\hline & \multicolumn{2}{c|}{ SW } & \multicolumn{2}{c|}{ EM } & \multicolumn{2}{c|}{$\mathrm{RE}$} & \multicolumn{2}{c}{$\mathrm{MT}$} \\
\cline { 2 - 8 } & $s 1$ & $s 2$ & $s 1$ & $s 2$ & $s 1$ & $s 2$ & $s 1$ & $s 2$ \\
\hline$t 1$ & 50 & 12 & 28 & -8 & 36 & 8 & 50 & 12 \\
$t 2$ & 50 & 50 & 50 & 10 & 45 & 3.5 & 50 & 50 \\
$t 3$ & 50 & 49 & -3 & -13 & 11 & -16.5 & 47 & 29 \\
\hline
\end{tabular}


Table 8: Load from each model(MW)

\begin{tabular}{|c|c|c|c|c|c|c|c|}
\hline & \multirow{3}{*}{ Time } & \multicolumn{2}{|c|}{ Schedule } & \multicolumn{4}{|c|}{ Operation } \\
\hline & & \multirow{2}{*}{$j 1$} & \multirow{2}{*}{$j 2$} & \multicolumn{2}{|c|}{$j 1$} & \multicolumn{2}{|c|}{$j 2$} \\
\hline & & & & $s 1$ & $s 2$ & $s 1$ & $s 2$ \\
\hline \multirow{3}{*}{ SW } & $t 1$ & 48 & 80 & 48 & 48 & 80 & 80 \\
\hline & $t 2$ & 30 & 84 & 30 & 30 & 84 & 84 \\
\hline & $t 3$ & 54 & 114 & 54 & 36 & 114 & 114 \\
\hline \multirow{3}{*}{ EM } & $t 1$ & 32 & 92 & 32 & 32 & 92 & 92 \\
\hline & $t 2$ & 30 & 96 & 30 & 30 & 96 & 96 \\
\hline & $t 3$ & 48 & 87 & 48 & 48 & 87 & 87 \\
\hline \multirow{3}{*}{$\mathrm{RN}$} & $t 1$ & 32 & 80 & 44 & 32 & 85 & 97 \\
\hline & $t 2$ & 20 & 64 & 30 & 26.5 & 96 & 94.5 \\
\hline & $t 3$ & 36 & 76 & 36 & 51.5 & 111 & 83.5 \\
\hline \multirow{3}{*}{ MT } & $t 1$ & 48 & 80 & 48 & 48 & 80 & 80 \\
\hline & $t 2$ & 30 & 82.5 & 30 & 30 & 82.5 & 82.5 \\
\hline & $t 3$ & 36 & 112.5 & 36 & 36 & 112.5 & 112.5 \\
\hline
\end{tabular}

Table 9: Wind power schedule and spillage (MW)

\begin{tabular}{ccccc|cc|cc|cc|cc}
\hline & \multicolumn{3}{c|}{ Wind Schedule } & \multicolumn{7}{c}{ Wind Spillage } \\
\cline { 2 - 12 } & SW & EM & RN & MT & \multicolumn{2}{|c}{ SW } & \multicolumn{2}{c}{ EM } & RN & \multicolumn{2}{c}{ MT } \\
& & & & $s 1$ & $s 2$ & $s 1$ & $s 2$ & $s 1$ & $s 2$ & $s 1$ & $s 2$ \\
\hline 1 & 32 & 26 & 13 & 32 & 0 & 0 & 0 & 0 & 0 & 0 & 0 & 0 \\
$t 2$ & 40 & 50 & 13 & 40 & 35 & 0 & 5 & 0 & 0 & 0 & 33 & 0 \\
$t 3$ & 35 & 25 & 0 & 35 & 0 & 0 & 0 & 0 & 0 & 0 & 0 & 0 \\
\hline
\end{tabular}

\subsubsection{Weights of the Comprehensive Model}

Since the proposed three single-objective models can be regarded as particular cases of the MT model, different weight combinations are possible. Weights $(0.2,0.3,0.5)$ above provides an example. To find out how weights affect the optimal scheduling results, every possible combination of weights with a step size of 0.04 are tested in this subsection.

Results are depicted in Fig. 2, where each dot represents an optimal solution under a set of weights. The weights are represented by the distance from the point to the corresponding edge of the equilateral triangle, as illustrated in Fig. 3. The color of the dot provides the relative value of the key performance indicators (KPIs), where blue means the lowest and red means the highest, as shown in Fig. 2(g). See 


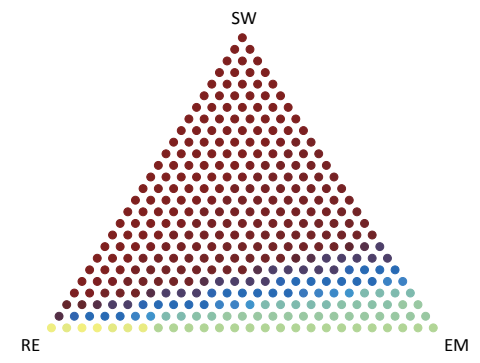

(a) Social Welfare

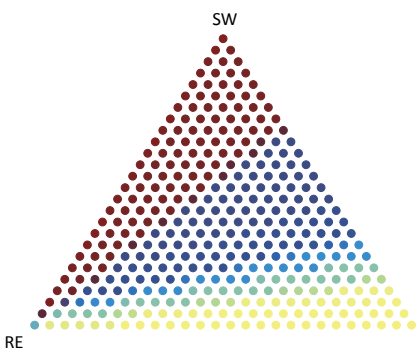

(d) Emissions

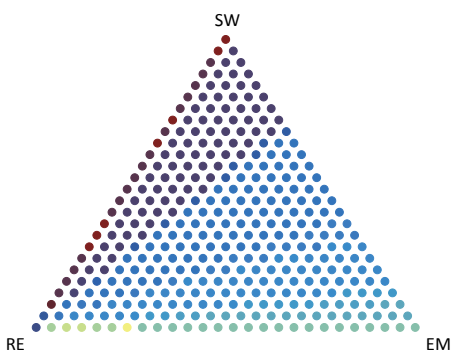

(b) Load Utility

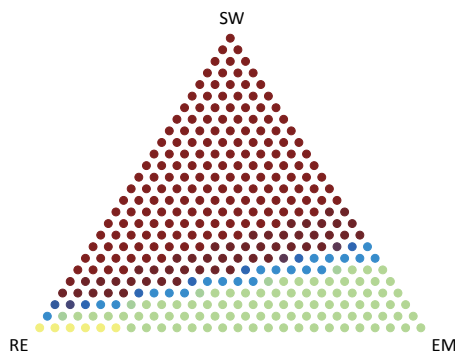

(e) Wind Spillage

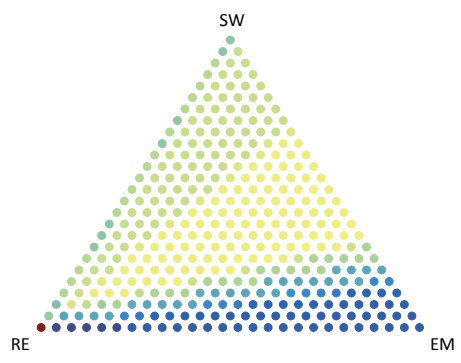

(c) Cost

Figure 2: KPIs under different weights.

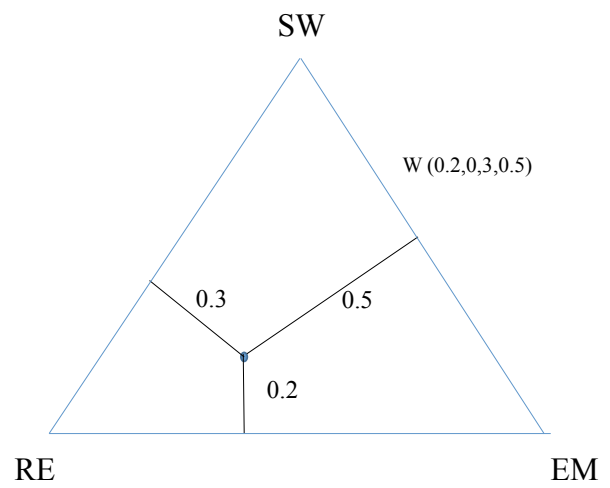

Figure 3: Illustration of the weights.

Table 5 for the ranges of actual values.

Optimal results under all the possible weights form the Pareto optimal set of the three objectives. Fig. 4 depicts the relationship among social welfare, cost and emission under different wind spillage levels. Allowing higher wind power spillage generally yields higher social welfare, but higher emissions as well. There is a non-trivial trade-off among wind power spillage, emissions, and social welfare. A medium level of wind power spillage is advisable to balance emissions and social welfare. Since generally there are multiple optimal solutions, especially for the 'zero wind spillage' cases, some points may deviate from the general trend. Using a multi-objective model with small weights on secondary objectives instead of single-objective models mitigates this issue. 


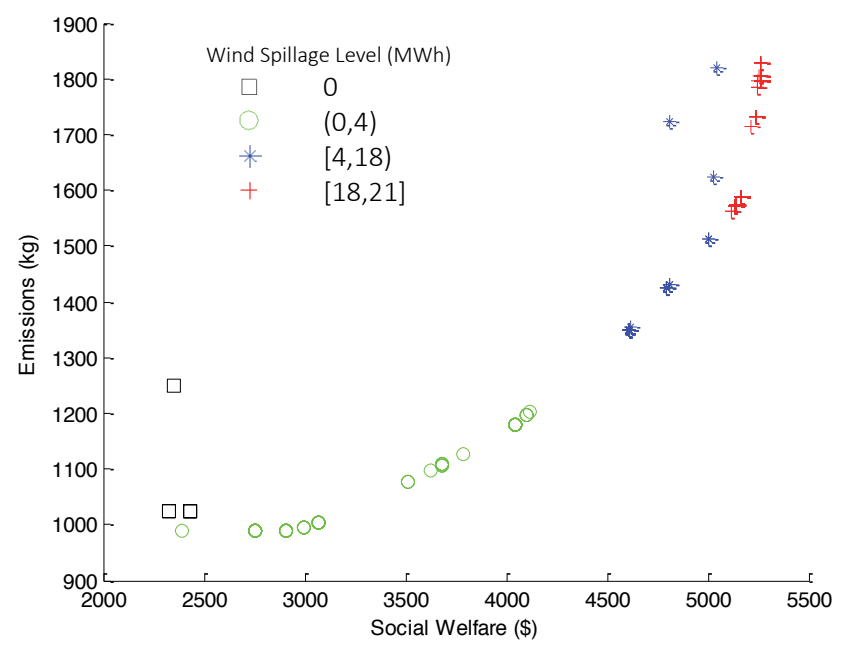

(a) Social Welfare vs. Emissions

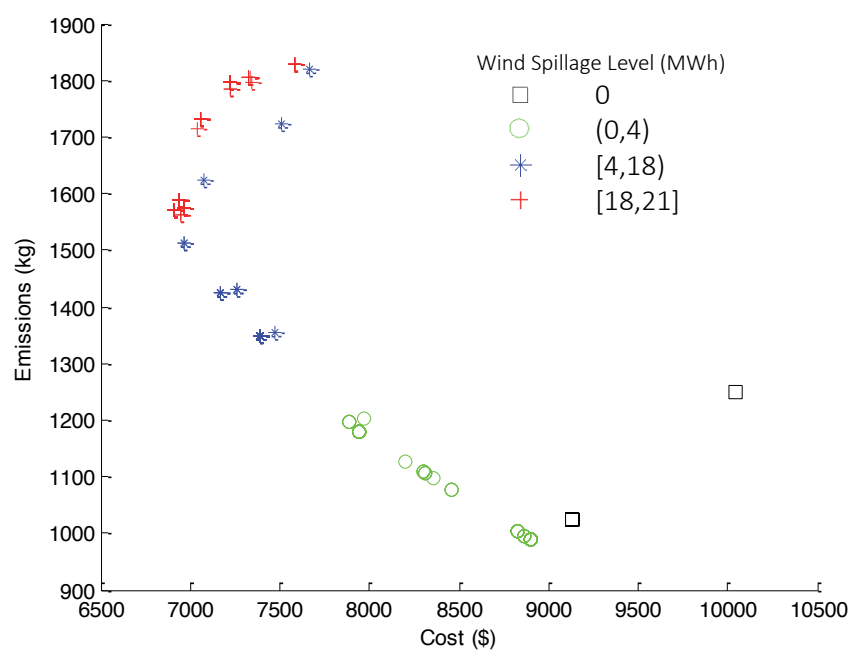

(b) Cost vs. Emissions

Figure 4: Social welfare, cost, emission and wind spillage.

Since the weights affect the optimization results, they should be carefully selected. From the perspective of goal planning, one may set the weights according to the KPIs of the optimization results and the expected KPIs. For example, if the system operator would like to keep the social welfare above a certain level, then an available area can be specified in Fig. 2(a). Similarly, another appropriate area can be selected in Fig. 2(d). A feasible set of weights can be obtained by overlapping the KPI triangles and taking the intersection of the available areas of each triangle. The corresponding values or weights are then set according to the government policies. 


\subsubsection{Data}

The IEEE 57-node system [34] is used to compare the proposed models. 24-hour load curves based on some areas of PJM on 7/30/2014 [35] are adopted. The peak load is 2,000 MW, and the valley load is 1,197 MW. The average marginal load utility in each hour is depicted in Fig. 5. The generation mix includes 10 coal-fired units, 6 gas-fired units and 1 wind unit. Parameters of the thermal units come from [36] and [37], and are provided in Table 10. The total thermal capacity is 3,229 MW. 10 wind production scenarios are generated using the model in [38].

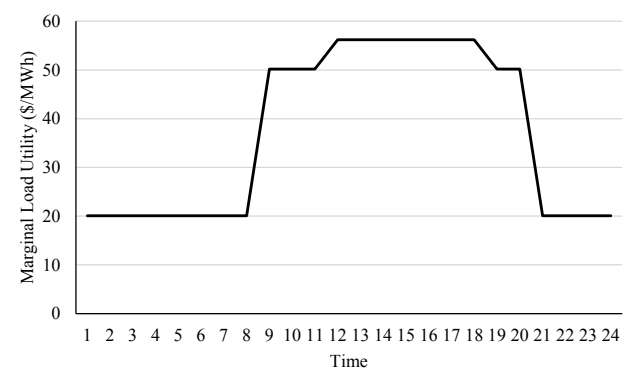

Figure 5: Average marginal demand utility.

Table 10: Data of thermal units

\begin{tabular}{|c|c|c|c|c|c|c|c|c|c|c|}
\hline Unit & Fuel & Node & $\begin{array}{l}P_{i}^{\max } \\
(\mathrm{MW})\end{array}$ & $\begin{array}{l}P_{i}^{\min } \\
(\mathrm{MW})\end{array}$ & $\begin{array}{c}C_{i} \\
(\$ / \mathrm{MWh})\end{array}$ & $\begin{array}{l}\pi_{i}^{\mathrm{SU}} \\
(\$)\end{array}$ & $\begin{array}{c}\sigma_{i}^{\mathrm{NO}_{\mathrm{x}}} \\
(\mathrm{kg} / \mathrm{MWh})\end{array}$ & $\begin{array}{c}\sigma_{i}^{\mathrm{SO}_{2}} \\
(\mathrm{~kg} / \mathrm{MWh})\end{array}$ & $\begin{array}{c}\sigma_{i}^{\mathrm{SU}, \mathrm{NO}_{\mathrm{x}}} \\
(\mathrm{kg})\end{array}$ & $\begin{array}{c}\sigma_{i}^{\mathrm{SU}, \mathrm{SO}_{2}} \\
(\mathrm{~kg})\end{array}$ \\
\hline$i 1$ & gas & $n 1$ & 49.3 & 10 & 47.9 & 478.7 & 0.404 & 0.003 & 0.404 & 0.003 \\
\hline$i 2$ & coal & $n 2$ & 403.7 & 200 & 24.3 & $1,166.9$ & 2.326 & 4.730 & 4.652 & 9.461 \\
\hline$i 3$ & coal & $n 3$ & 236 & 140 & 25.2 & $1,208.2$ & 1.499 & 1.181 & 11.990 & 2.363 \\
\hline$i 4$ & coal & $n 6$ & 200 & 56 & 26.2 & $1,259.5$ & 2.459 & 8.810 & 4.918 & 17.621 \\
\hline$i 5$ & coal & $n 8$ & 299.2 & 110 & 21.7 & $1,041.7$ & 1.997 & 12.818 & 3.993 & 25.637 \\
\hline$i 6$ & gas & $n 9$ & 170 & 111 & 47.0 & 469.8 & 0.165 & 0.003 & 0.165 & 0.003 \\
\hline$i 7$ & gas & $n 12$ & 107 & 6 & 55.6 & 556.0 & 0.410 & 0.004 & 0.410 & 0.004 \\
\hline$i 8$ & coal & $n 13$ & 125 & 50 & 25.6 & $1,230.6$ & 0.898 & 0.365 & 1.796 & 0.731 \\
\hline$i 9$ & coal & $n 17$ & 125 & 50 & 25.2 & $1,210.6$ & 1.036 & 0.293 & 2.072 & 0.586 \\
\hline$i 10$ & coal & $n 19$ & 725.9 & 225 & 25.4 & $1,221.5$ & 0.775 & 0.650 & 1.549 & 1.301 \\
\hline$i 11$ & coal & $n 23$ & 81.6 & 40 & 25.8 & $1,236.2$ & 0.975 & 3.554 & 1.951 & 7.108 \\
\hline$i 12$ & coal & $n 29$ & 163.2 & 65 & 23.4 & $1,122.2$ & 3.396 & 11.402 & 6.792 & 22.803 \\
\hline$i 13$ & gas & $n 31$ & 121 & 65 & 52.7 & 526.8 & 0.210 & 0.004 & 0.210 & 0.004 \\
\hline$i 14$ & gas & $n 37$ & 173.4 & 95 & 32.4 & 323.9 & 0.036 & 0.002 & 0.144 & 0.002 \\
\hline$i 15$ & gas & $n 43$ & 173.4 & 95 & 31.3 & 313.5 & 0.040 & 0.002 & 0.161 & 0.002 \\
\hline$i 16$ & coal & $n 53$ & 75 & 25 & 24.7 & $1,186.5$ & 0.722 & 2.385 & 1.444 & 4.770 \\
\hline
\end{tabular}




\subsubsection{Results}

The summary of the simulation results is provided in Table 11. Model SW results in the highest social welfare, the highest demand utility, the lowest cost, the highest demand supplied, and the highest emissions. Model EM has the lowest demand supplied to reduce emission. Compared with model SW, model EM results in $73 \%$ reduction in emissions, but a reduction in social welfare of $64 \%$. Wind spillage in model EM is lower than that in model SW. Model RE results in the lowest wind spillage. The social welfare and emissions range from those from model SW and those from model EM. Model MT, with the weights $\left(w_{\mathrm{SW}}, w_{\mathrm{EM}}, w_{\mathrm{RE}}\right)=(0.05,0.4,0.55)$, provides intermediate results.

Table 11: Key Performance Indicators using each model

\begin{tabular}{cccccccccc}
\hline & Social & Demand & Cost & Emissions & Wind & Demand & Solution & Relative \\
Model & Welfare & Utility & & & Spillage & Supplied & Time & Gap \\
& $(\$)$ & $(\$)$ & $(\$)$ & $(\mathrm{kg})$ & $(\mathrm{MWh})$ & $(\mathrm{MWh})$ & $(\mathrm{sec})$ & \\
\hline SW & 840,555 & $1,782,032$ & 941,477 & 60,449 & 1,191 & 40,686 & 14 & 0.00055 \\
EM & 299,135 & $1,538,529$ & $1,239,394$ & 16,196 & 516 & 39,315 & 601 & 0.00003 \\
RE & 462,332 & $1,658,018$ & $1,195,685$ & 34,048 & 339 & 39,992 & 494 & 0.00086 \\
MT & 782,410 & $1,766,647$ & 984,238 & 33,376 & 679 & 40,263 & 61 & 0.00067 \\
\hline
\end{tabular}

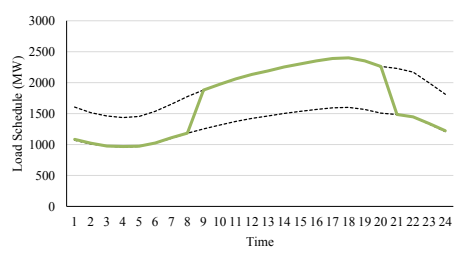

(a) SW

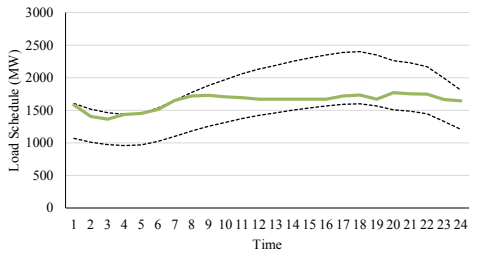

(b) EM

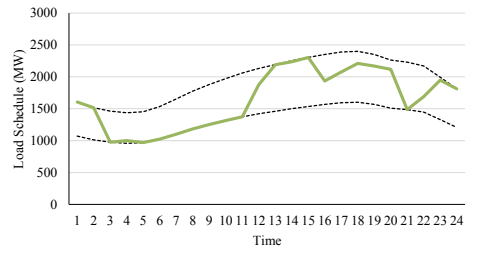

(c) RE

Figure 6: Scheduled demand supply: SW vs. EM vs. RE.

The scheduled demand from each model is depicted in Fig. 6, where the dash lines are the maximum and minimum dispatchable loads, and the solid lines are demand supplied at schedule level. Dispatchable loads play an important role to achieve the goal in each optimization model. In model SW, load moves up and down with the marginal utility to maximize social welfare. Since the marginal emission goes up with the load, a flat load curve is good for low emissions while complying with the minimum energy consumption constraint. Using model RE, the load adapts to wind production to accommodate more and more renewable energy.

Fig. 7 depict the generation scheduling in the three models by energy type. The cheap coal-fired units produce more energy if model SW is used, while cleaner gas-fired units produce at their maximum if model EM is used. Coal- and gas-fired units as well as the dispatchable loads coordinate to accommodate wind power if model RN is used. Note that the scheduled wind power in (5c) is usually used to get price [39], 


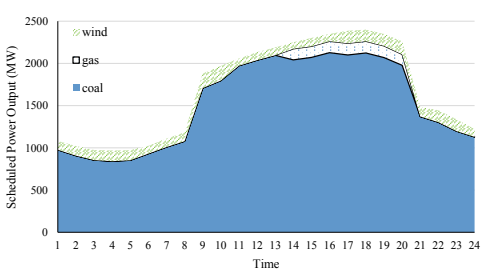

(a) SW

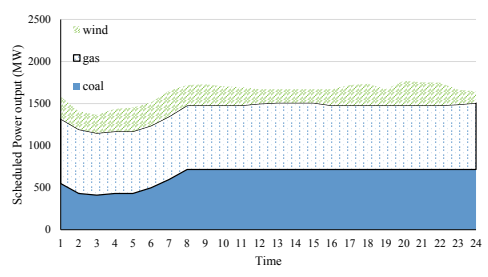

(b) EM

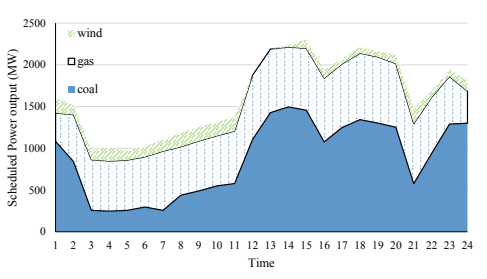

(c) RE

Figure 7: Power outputs: SW vs. EM vs. RE.

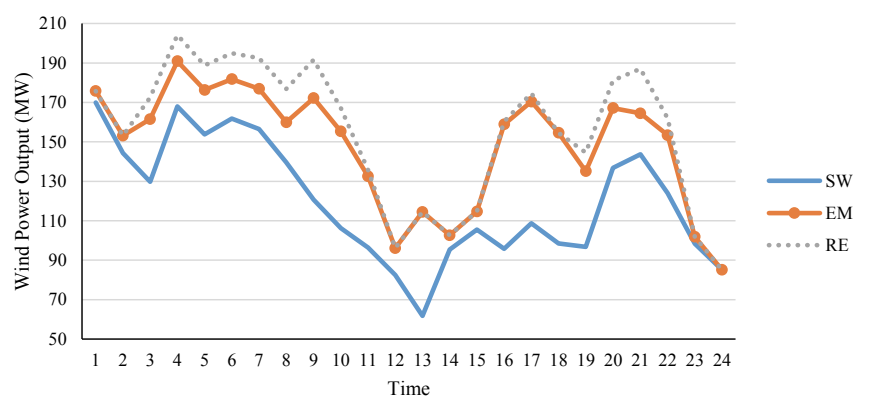

Figure 8: Average wind power production.

and it is different from the actual wind production (see Fig. 8).

The models are coded in GAMS [40], solved by CPLEX 12.6 and implemented on a laptop with 2.5 $\mathrm{GHz}$ CPU and $16 \mathrm{~GB}$ of RAM. The models have 61,921 equations, 1,152 binary variables and 89,086 continuous variables. The calculation time and accuracy are listed in Table 11. They may vary among different objectives.

\section{Conclusions}

This paper compares three stochastic generation scheduling models that seek to maximize social welfare, to minimize emissions, or to minimize wind power spillage. The proposed framework allows analyzing generation scheduling results under different policies, particularly those related to the Chinese government. Conclusions are listed below:

1. Model SW results in the highest social welfare, attained by increasing demand utility and reducing cost. Specifically, the flexible load increases at high utility hours. Model SW may lead to 3.7 times higher emissions and 3.5 times higher wind spillage than the emissions and wind spillage of models EM and RE, respectively.

2. Model EM results in $27 \%$ of the emissions of the SW model, $36 \%$ of its social welfare, $132 \%$ of its cost, $97 \%$ of its demand supplied and $43 \%$ of its wind spillage. 
3. Model RE results in $56 \%$ of the emissions of the SW model, $55 \%$ of its social welfare, $126 \%$ of its cost, $98 \%$ of its demand supplied and $28 \%$ of its wind spillage. Note that the demand adapts to variations of wind power production in this model.

4. Model MT provides balanced results as compared to any of the single objective models. It results in $93 \%$ of the social welfare of the SW model, $55 \%$ of its emissions and $57 \%$ of its wind spillage.

5. The weights of model MT, set according to system operator's or government's policies, results in an optimal generation scheduling that balances the objectives. A method to set appropriate weights according to KPI targets is proposed.

6. It is sensible to conclude the following: In highly polluted and populated regions, the weight of the emission term should be comparatively high. In power systems with significant wind power, the weight of the wind spillage term should be comparatively high. Generally, weights should be chosen carefully since their marginal impact decreases as emissions or wind spillage decreases, or social welfare increases.

Finally, we point out that this paper proposes a method to analyze scheduling models with different objectives. Specific conclusions generally depend on the characteristics of the power system under study.

\section{Acknowledgments}

The authors would like to thank the following funding supports. Z. Geng is partly supported by China Scholarship Council. A. J. Conejo is partly supported by NSF award 1548015. Q. Chen is partly supported by the NSF of China grant 51622705, Fok Ying Tung Education Foundation award 151057 and Beijing New-star Plan of Science and Technology. Q. Xia is partly supported by the NSF of China grant 51537005. C. Kang is partly supported by the NSF of China grant 51325702 .

\section{References}

[1] Environmental statistics yearbook 2014.

URL http://zls.mep.gov.cn/hjtj/nb/

[2] J. I. Lewis, Wind energy in China: Getting more from wind farms, Nature Energy 1 (2016) 1-2. doi:10.1038/NENERGY.2016.76.

[3] J. Soares, M. A. Fotouhi Ghazvini, Z. Vale, P. B. de Moura Oliveira, A multi-objective model for the day-ahead energy resource scheduling of a smart grid with high penetration of sensitive loads, Applied Energy 162 (2016) 1074-1088. doi:10.1016/j .apenergy.2015.10.181. 
[4] J. Wang, M. Shalidehpour, Z. Li, Security-constrained unit commitment with volatile wind power generation, IEEE Transactions On Power Systems 23 (3) (2008) 1319-1327. doi:10.1109/TPWRS. 2008.926719.

[5] B. Jiang, A. M. Farid, K. Youcef-Toumi, Demand side management in a day-ahead wholesale market: A comparison of industrial \& social welfare approaches, Applied Energy 156 (2015) 642-654. doi: $10.1016 / j$. apenergy $\cdot 2015 \cdot 07.014$.

[6] M. R. Gent, J. W. Lamont, Minimum-emission dispatch, IEEE Transactions on Power Apparatus and Systems PAS-90 (6) (1971) 2650-2660. doi:10.1109/TPAS.1971.292918.

[7] A. Nasri, A. J. Conejo, S. J. Kazempour, M. Ghandhari, Minimizing wind power spillage using an opf with FACTS devices, IEEE Transactions on Power Systems 29 (5) (2014) 2150-2159. doi:10.1109/ TPWRS. 2014.2299533.

[8] A. Glotic, A. Zamuda, Short-term combined economic and emission hydrothermal optimization by surrogate differential evolution, Applied Energy 141 (2015) 42-56. doi:10.1016/j.apenergy . 2014 . 12.020 .

[9] M. Reza Norouzi, A. Ahmadi, A. Esmaeel Nezhad, A. Ghaedi, Mixed integer programming of multiobjective security-constrained hydro/thermal unit commitment, Renewable and Sustainable Energy Reviews 29 (2014) 911-923. doi:10.1016/j.rser.2013.09.020.

[10] A. Ahmadi, M. Sharafi Masouleh, M. Janghorbani, N. Yadollahi Ghasemi Manjili, A. M. Sharaf, A. Esmaeel Nezhad, Short term multi-objective hydrothermal scheduling, Electric Power Systems Research 121 (2015) 357-367. doi:10.1016/j.epsr.2014.11.015.

[11] H. R. Sadeghian, M. M. Ardehali, A novel approach for optimal economic dispatch scheduling of integrated combined heat and power systems for maximum economic profit and minimum environmental emissions based on benders decomposition, Energy 102 (2016) 10-23. doi:10.1016/j . energy . 2016. 02.044 .

[12] T. Niknam, R. Azizipanah-Abarghooee, M. R. Narimani, An efficient scenario-based stochastic programming framework for multi-objective optimal micro-grid operation, Applied Energy 99 (2012) 455-470. doi:10.1016/j. apenergy.2012.04.017.

[13] A. Zakariazadeh, S. Jadid, P. Siano, Economic-environmental energy and reserve scheduling of smart distribution systems: A multiobjective mathematical programming approach, Energy Conversion and Management 78 (2014) 151-164. doi:10.1016/j.enconman.2013.10.051.

[14] H. Falsafi, A. Zakariazadeh, S. Jadid, The role of demand response in single and multi-objective wind-thermal generation scheduling: A stochastic programming, Energy 64 (2014) 853-867. doi: 10.1016/j . energy . 2013.10.034. 
[17] H. C. Sampaio, R. A. Dias, J. A. P. Balestieri, Sustainable urban energy planning: The case study of a tropical city, Applied Energy 104 (2013) 924-935. doi:10.1016/j.apenergy .2012.12.022.

[18] V. M. Charitopoulos, V. Dua, A unified framework for model-based multi-objective linear process and energy optimisation under uncertainty, Applied Energy 186 (2017) 539-548. doi:10.1016/j.

[19] A. Trivedi, D. Srinivasan, D. Sharma, C. Singh, Evolutionary multi-objective day-ahead thermal generation scheduling in uncertain environment, IEEE Transactions on Power Systems 28 (2) (2013) 1345-1354. doi:10.1109/TPWRS.2012.2222939.

[20] F.-F. Li, J. Qiu, Multi-objective optimization for integrated hydro?photovoltaic power system, Applied

[21] K. Deb, A. Pratap, S. Agarwal, T. Meyarivan, A fast and elitist multiobjective genetic algorithm: Nsga-ii, IEEE Transactions on Evolutionary Computation 6 (2) (2002) 182-197. doi:10.1109/4235. 996017.

[22] M. A. Hozouri, A. Abbaspour, M. Fotuhi-Firuzabad, M. Moeini-Aghtaie, On the use of pumped storage for wind energy maximization in transmission-constrained power systems, IEEE Transactions on Power Systems 30 (2) (2015) 1017-1025. doi:10.1109/TPWRS.2014.2364313.

[23] Q. P. Zheng, J. Wang, A. L. Liu, Stochastic optimization for unit commitment-a review, IEEE Transactions on Power Systems 30 (4) (2015) 1913-1924. doi:10.1109/TPWRS. 2014. 2355204.

[24] T. Schulze, K. McKinnon, The value of stochastic programming in day-ahead and intra-day generation unit commitment, Energy 101 (2016) 592-605. doi:10.1016/j.energy .2016.01.090.

[25] J. Wang, A. Botterud, R. Bessa, H. Keko, L. Carvalho, D. Issicaba, J. Sumaili, V. Miranda, Wind power forecasting uncertainty and unit commitment, Applied Energy 88 (11) (2011) 4014-4023. doi: 10.1016/j.apenergy.2011.04.011.

[26] J. Wang, J. Wang, C. Liu, J. P. Ruiz, Stochastic unit commitment with sub-hourly dispatch constraints, Applied Energy 105 (2013) 418-422. doi:10.1016/j.apenergy.2013.01.008. 
[27] R. Dominguez, A. J. Conejo, M. Carrion, Operation of a fully renewable electric energy system with csp plants, Applied Energy 119 (2014) 417-430. doi:10.1016/j.apenergy .2014.01.014.

[28] J. M. Morales, A. J. Conejo, J. Perez-Ruiz, Economic valuation of reserves in power systems with high penetration of wind power, IEEE Transactions on Power Systems 24 (2) (2009) 900-910. doi: 10.1109/TPWRS. 2009.2016598.

[29] A. J. Conejo, M. Carrion, J. Morales, Decision making under uncertainty in electricity markets. International series in Operations Research \& Management Science, Springer, New York, NY, 2010. doi:10.1007/978-1-4419-7421-1.

[30] J. M. Morales, A. J. Conejo, H. Madsen, P. Pinson, M. Zugno, Integrating renewables in electricity markets: Operational problems, Springer, New York, NY, 2013. doi:10.1007/978-1-4614-9411-9.

[31] Z. Mi, Y.-M. Wei, B. Wang, J. Meng, Z. Liu, Y. Shan, J. Liu, D. Guan, Socioeconomic impact assessment of China's $\mathrm{CO}_{2}$ emissions peak prior to 2030, Journal of Cleaner Production 142 (2017) 2227-2236. doi:10.1016/j.jclepro.2016.11.055.

[32] Y.-M. Wei, Z.-F. Mi, Z. Huang, Climate policy modeling: An online SCI-E and SSCI based literature review, Omega 57 (2015) 70-84. doi:10.1016/j.omega.2014.10.011.

[33] IBM ILOG CPLEX Optimization Studio.

URL https://www-01.ibm.com/software/commerce/optimization/cplex-optimizer/

[34] Power systems test case archive.

URL http://www.ee.washington.edu/research/pstca/

[35] PJM metered load data.

URL http://www.pjm.com/markets-and-operations/ops-analysis/historical-load-data. aspx

[36] Electricity form EIA-860 2014.

URL http://www.eia.gov/electricity/data/eia860/xls/eia8602014.zip

$415 \quad[37]$ Air markets program data.

URL http://ampd.epa.gov/ampd/

[38] System advisor model.

URL https://sam.nrel.gov/

[39] J. M. Morales, A. J. Conejo, K. Liu, J. Zhong, Pricing electricity in pools with wind producers, IEEE Transactions on Power Systems 27 (3) (2012) 1366-1376. doi:10.1109/TPWRS.2011.2182622.

[40] General algebraic modeling system (GAMS).

URL https://www.gams.com/ 